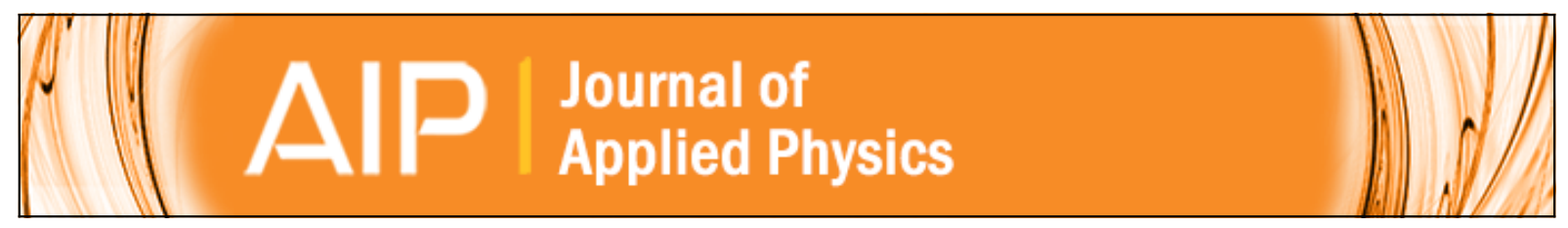

\title{
Influence of the demagnetizing field on the determination of the magnetocaloric effect from magnetization curves
}

R. Caballero-Flores, V. Franco, A. Conde, and L. F. Kiss

Citation: Journal of Applied Physics 105, 07 A919 (2009); doi: 10.1063/1.3067463

View online: http://dx.doi.org/10.1063/1.3067463

View Table of Contents: http://scitation.aip.org/content/aip/journal/jap/105/7?ver=pdfcov

Published by the AIP Publishing

\section{Articles you may be interested in}

Effect of hydrostatic pressure on magnetic entropy change and critical behavior of the perovskite manganite La0.4Bi0.3Sr0.3MnO3

J. Appl. Phys. 113, 023904 (2013); 10.1063/1.4774107

Influence of $\mathrm{Co}$ and $\mathrm{Ni}$ addition on the magnetocaloric effect in $\mathrm{Fe} 88-2 \times \mathrm{Co} \times \mathrm{Ni} \times \mathrm{Zr} 7 \mathrm{~B} 4 \mathrm{Cu} 1$ soft magnetic amorphous alloys

Appl. Phys. Lett. 96, 182506 (2010); 10.1063/1.3427439

Magnetocaloric response of $\mathrm{FeCrB}$ amorphous alloys: Predicting the magnetic entropy change from the Arrott-Noakes equation of state

J. Appl. Phys. 104, 033903 (2008); 10.1063/1.2961310

Field dependence of the magnetocaloric effect in materials with a second order phase transition: A master curve for the magnetic entropy change

Appl. Phys. Lett. 89, 222512 (2006); 10.1063/1.2399361

Analysis of magnetization and magnetocaloric effect in amorphous FeZrMn ribbons

J. Appl. Phys. 97, 10M310 (2005); 10.1063/1.1853193

\section{SHIMADzU Powerful, Multi-functional UV-Vis-NIR and Excellence in Science FTIR Spectrophotometers}

Providing the utmost in sensitivity, accuracy and resolution for applications in materials characterization and nano research

- Photovoltaics

- Polymers

- Thin films

- Paints

- Ceramics

- DNA film structures

- Coatings

- Packaging materials
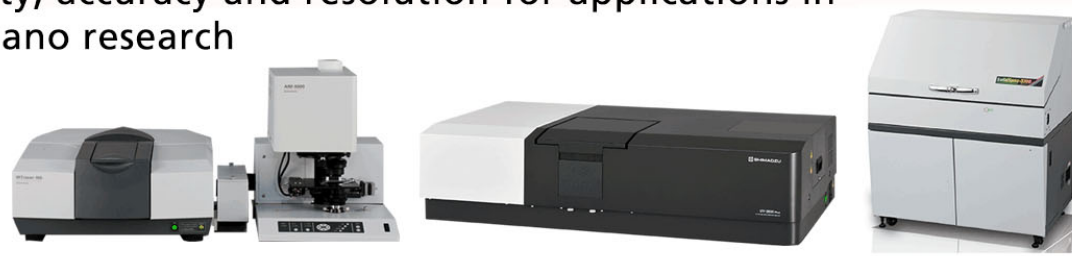


\title{
Influence of the demagnetizing field on the determination of the magnetocaloric effect from magnetization curves
}

\author{
R. Caballero-Flores, ${ }^{1}$ V. Franco, ${ }^{1, a)}$ A. Conde, ${ }^{1}$ and L. F. Kiss ${ }^{2}$ \\ ${ }^{1}$ Dpto. Física de la Materia Condensada, ICMSE-CSIC, Universidad de Sevilla, P.O. Box 1065, \\ 41080 Sevilla, Spain \\ ${ }^{2}$ Research Institute for Solid State Physics and Optics, Hungarian Academy of Sciences, P.O. Box 49, \\ 1525 Budapest, Hungary
}

(Presented 14 November 2008; received 20 August 2008; accepted 2 November 2008; published online 12 February 2009)

The influence of the demagnetizing factor $(N)$ on the magnetic entropy change $\left(\Delta S_{M}\right)$ curves is studied for materials with a second order phase transition. For this purpose, a soft magnetic amorphous ribbon is measured for different orientations of the magnetic field with respect to the plane of the sample. For temperatures below the Curie temperature $\left(T_{C}\right)$, the increase in $N$ causes a decrease in $\Delta S_{M}$, while for temperatures above $T_{C}$ no change in the shape of the curves has been found for the different orientations, as expected. In order to eliminate this influence of $N$ and compare the $\Delta S_{M}(T)$ curves for samples with different shapes, the recently proposed universal curve for the magnetocaloric effect can be used. (C) 2009 American Institute of Physics.

[DOI: $10.1063 / 1.3067463$ ]

The recent increase in the research on magnetocaloric effect (MCE) is due to its promising application for magnetic refrigeration at room temperature. It could allow more efficient and environmental friendly systems than those based on the conventional gas compression-expansion cycle. Although the larger MCE response corresponds to materials with a first order magneto-structural phase transition (the socalled giant magnetocaloric effect $\left.{ }^{1}\right)$, most of the current refrigerator prototypes still use materials with a second order phase transition due to the lack of thermal hysteresis, ${ }^{2}$ being Gd a paradigmatic case. In the literature, it is usual to disregard the value of the demagnetizing factor to calculate the magnetic entropy change from magnetization curves. In the case of ribbon shaped samples measured with the applied field contained in the plane of the ribbon, the influence of the demagnetizing factor $N$ can be neglected due to the large aspect ratio. However, this is not the typical case in the study of magnetocaloric materials. The aim of this work is twofold: on the one hand, to analyze the influence of $N$ on the temperature dependence of $\Delta S_{M}$, and on the other hand, to find a method to compare results obtained from samples with different shapes.

An amorphous ribbon of $\mathrm{Fe}_{77} \mathrm{Cr}_{8} \mathrm{~B}_{15}$ composition ( $1 \mathrm{~mm}$ wide and $\sim 20 \mu \mathrm{m}$ thick) was obtained by melt spinning. The amorphous character of the as-quenched alloy was checked by x-ray diffraction. The field (up to $15 \mathrm{kOe}$ ) and temperature $(300-500 \mathrm{~K})$ dependence of magnetization $(M)$ of $3 \mathrm{~mm}$ long samples have been measured in a vibrating sample magnetometer with different orientations of the applied magnetic field with respect to the plane of the sample. More details about the microstructural and magnetic characterization of the sample are given elsewhere. ${ }^{3}$ Rotation of the sample around an axis perpendicular to the ribbon plane

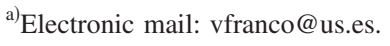

gives no evidence in the magnetization curves of neither a preferential magnetization axis contained in the plane of the ribbon, nor of an out of plane easy axis. Taking into account the amorphous character of the sample, together with its extreme magnetic softness, we can assume that the main contribution to the magnetic anisotropy is determined by shape. The magnetic entropy change due to the application of a magnetic field $H$ has been evaluated using a numerical approximation to the equation

$$
\Delta S_{M}=\int_{0}^{H}\left(\frac{\partial M}{\partial T}\right)_{H} d H
$$

where the partial derivative is replaced by finite differences and the integration is performed numerically.

Recently, a phenomenological universal curve for the magnetic entropy change has been proposed ${ }^{4}$ as a method to compare the properties of different materials and to make extrapolations to fields and/or temperatures outside the available experimental range. It is constructed by normalizing the magnetic entropy change curves with respect to their peak $\Delta S_{M}^{p k}$ and rescaling the temperature axis. Initially two different reference temperatures were used for scaling:

$$
\theta(2 \mathrm{ref})=\left\{\begin{array}{cc}
-\left(T-T_{C}\right) /\left(T_{r 1}-T_{C}\right) & T \leq T_{C}, \\
\left(T-T_{C}\right) /\left(T_{r 2}-T_{C}\right) & T>T_{C},
\end{array}\right.
$$

where $T_{r 1}$ and $T_{r 2}$ are the temperatures of the two reference points of each curve and $T_{C}$ is the Curie temperature. However, it has been recently shown theoretically ${ }^{5}$ and confirmed experimentally ${ }^{6}$ that it should be enough to use a single reference temperature $T_{r}$,

$$
\theta(1 \mathrm{ref})=\left(T-T_{C}\right) /\left(T_{r}-T_{C}\right) .
$$

The reference temperatures have been selected as the temperatures corresponding to $\Delta S_{M}\left(T_{r}\right) / \Delta S_{M}^{p k}=0.5$. For the 


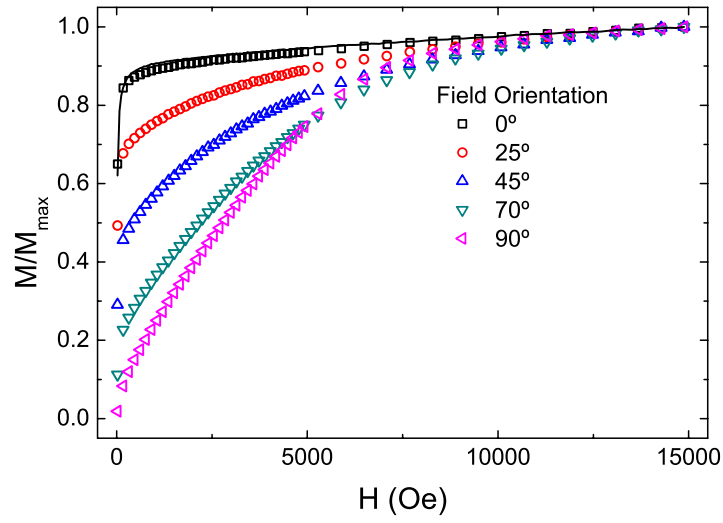

FIG. 1. (Color online) Field dependence of the room temperature magnetization of the sample with the magnetic field applied at different orientations with respect to the plane of the ribbon, rotating the sample around an axis contained in its plane $\left(0^{\circ}\right.$ corresponds to the field in plane; $90^{\circ}$ to the field perpendicular to the plane). Continuous line corresponds to the rotation of the sample around an axis perpendicular to the plane of the sample.

present study the Curie temperature has been identified as the temperature of the peak entropy change $T_{p k}$.

Figure 1 shows the field dependence of the room temperature magnetization of samples with different orientations with respect to the applied magnetic field. While there is no detectable influence of the orientation of the field when it is contained in the plane of the sample, the application of $H$ at different angles shows a tilting of the magnetization curve due to shape anisotropy. The small differences between the continuous line (sample on a bottom mount sample holder rotated around an axis perpendicular to its plane) and the $0^{\circ}$ curve (sample on a side mount sample holder with the field contained in plane) are due to the experimental uncertainties.

In order to make an accurate comparison between the magnetic entropy change curves, it should be taken into account that there could be a minor influence of small off-axis rotation of the sample, which alters the saddle point, slightly affects the moment value and, consequently, the calculated value of $\left|\Delta S_{M}\right|$. Therefore, it is preferable to normalize the curves to analyze the influence of the orientation of $H$ (Fig. 2 ). While above $T_{C}$ the differences between curves are inside the experimental error, at low temperatures there is a systematic decrease in $\left|\Delta S_{M}\right|$ as the demagnetizing factor $N$ increases.

The construction of the universal curve is a more adequate method for analyzing the influence of $N$. Figure 3 shows the rescaled magnetic entropy change curves for different maximum applied fields using a single reference temperature. Two different orientations of the field are presented: in plane $(N \approx 0)$ and perpendicular to the plane of the sample $(N \approx 1)$. While in the first case the collapse of the experimental data onto a single rescaled curve can be achieved, this is not the case for the perpendicular orientation. Increasing the maximum applied field in this second case produces an increase in the reduced magnetic entropy change for the same value of the reduced temperature. However, this increase is not homogeneous: as field is increased, the separation between the different curves is smaller.

The explanation of the low temperature evolution of $\Delta S_{M} / \Delta S_{M}^{p k}$ should be related to the fact that for higher de-

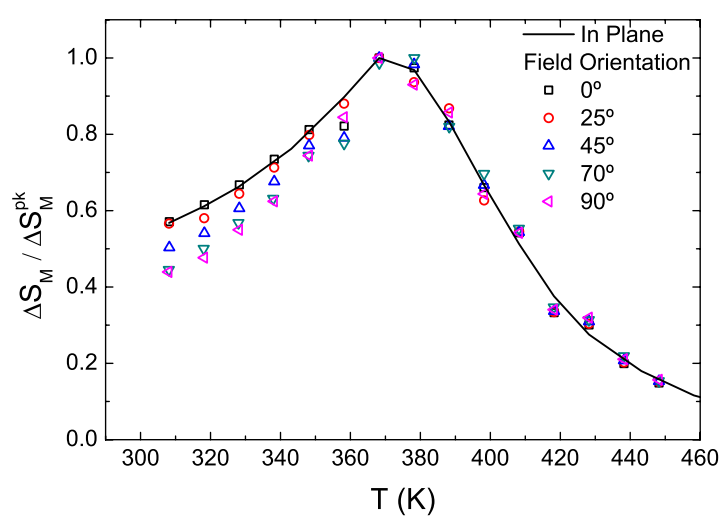

FIG. 2. (Color online) Influence of the orientation of the field on the normalized magnetic entropy change calculated from the magnetization data for a maximum applied field of $15 \mathrm{kOe}$. Symbols correspond to the rotation of the sample around an axis contained in the plane of the sample (field forming an angle with respect to the ribbon plane); continuous line corresponds to the rotation around an axis perpendicular to the plane of the ribbon (field in plane).

magnetizing factors, the field required to saturate the sample is larger, as seen in Fig. 1. Let us consider that the magnetic equation of state of a ferromagnetic material in the proximity of the transition temperature can be approximately described by the Arrott-Noakes equation of state, ${ }^{7}$ which can be written as

$$
H^{1 / \gamma}=a\left(T-T_{C}\right) M^{1 / \gamma}+b M^{1 / \beta+1 / \gamma},
$$

where $\beta$ and $\gamma$ are the critical exponents. After proper manipulation of the equation of state, Eq. (1) can be rewritten as

$$
\Delta S_{M}=-\int_{M_{S}}^{M} a \gamma M\left(a\left(T-T_{C}\right)+b M^{1 / \beta}\right)^{\gamma-1} d M,
$$

where $M_{s}$ stands for the spontaneous magnetization and the upper limit of the integral is the magnetization corresponding to the maximum applied field at the given temperature. For a general case this expression cannot be simplified. In the case of a mean field model the values of the critical exponents $(\beta=0.5 ; \gamma=1)$ reduce it to

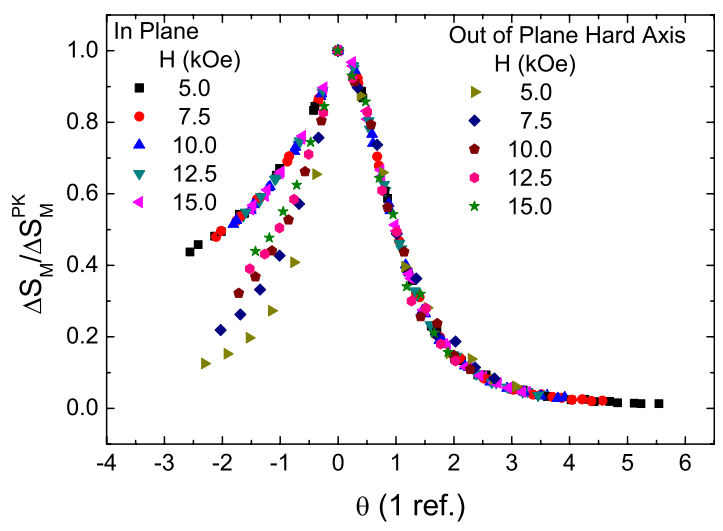

FIG. 3. (Color online) Rescaled magnetic entropy change data using a single reference temperature for different maximum field values applied in the plane of the ribbon and perpendicular to this plane. 


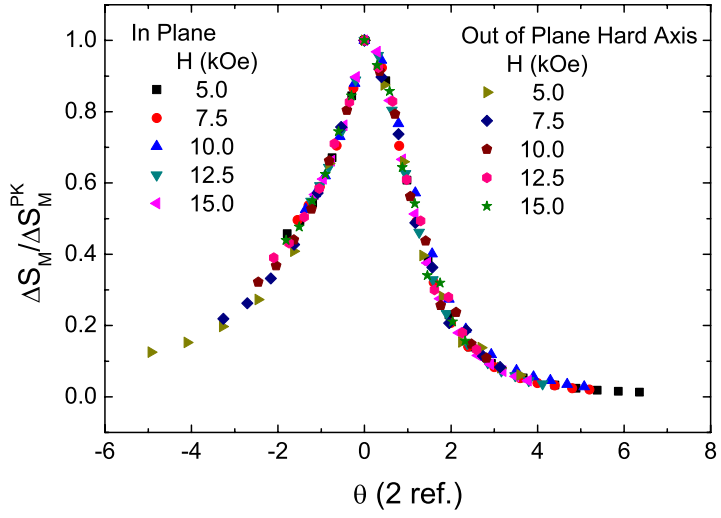

FIG. 4. (Color online) Rescaled magnetic entropy change data using two reference temperatures for different maximum field values applied in the plane of the ribbon and perpendicular to this plane.

$$
\Delta S_{M}(\text { mean field })=-\frac{1}{2} a\left(M^{2}-M_{s}^{2}\right) .
$$

The equation of state should be applicable to materials in technical saturation. However, for low applied fields and temperatures below $T_{C}$, both domain wall movement and magnetization rotation should be taken into account, and the experimental value of magnetization $M_{\exp }$ is smaller than $M$ predicted by Eq. (4). Therefore, Eq. (6) can be used to justify the decrease in $\Delta S_{M} / \Delta S_{M}^{p k}$ with increasing $H$ angle: for field perpendicular to the plane, the value of $M_{\exp }^{2}$ for moderate field values is much smaller than when the field is applied in plane, making $\Delta S_{M} / \Delta S_{M}^{p k}$ smaller. As $H$ increases, the difference between $M_{\exp }$ and $M$ decreases, leading to the finding that the separation between reduced magnetic entropy change curves is smaller for larger field values. For large enough field values (or for smaller angles of the applied field), the rescaled curve tends to that of the sample measured with the field in plane, in agreement with the trend observed in Fig. 2 for the angular dependence of the curve.

Although rescaling $\Delta S_{M} / \Delta S_{M}^{p k}$ curves with the use of a single reference temperature is not able to eliminate the influence of the demagnetizing factor, Fig. 4 shows that when the temperature axis is rescaled with the use of Eq. (2), all the experimental data points for the different orientations of the field collapse onto the same curve. In this case, to make more evident the quality of the collapse at the tails of the curves, the reference temperatures have been selected as $\Delta S_{M}\left(T_{r}\right) / \Delta S_{M}^{p k}=0.6$, with $T_{r 1}$ the one fulfilling that condition for each curve below $T_{C}$, and $T_{r 2}$ above it (this is a more demanding test than a factor of 0.5 , as the possible deviations would be magnified). The reason for this collapse is that although the field dependence of the magnetic entropy change is distorted from what is expected from the equation of state, this change does not have a strong temperature dependence except for temperatures close to the peak, where $N$ does not play an important role. Therefore, the second reference temperature can approximately account for this distortion below $T_{C}$, allowing the collapse of the experimental data.

In conclusion, the demagnetizing factor has an influence in the low temperature tails of the magnetic entropy change, narrowing the peak and affecting the calculated value of the refrigerant capacity for samples with different shapes $(R C$ decreases with increasing $N$ ). In order to compare experimental results from samples with different shapes, the universal curve constructed with two reference temperatures can be used, eliminating the influence of $N$ on the experimental data.

This work was supported by the Spanish Ministry of Science and Innovation and EU FEDER (Project Nos. MAT 2007-65227 and CIT-420000-2008-9), the PAI of the Regional Government of Andalucía (Project No. P06-FQM01823), the Hungarian-Spanish Academic Exchange Program for 2007-2008 (MTA-CSIC, Project No. 04; 2006HU0015), and the Hungarian Research Fund (Contract No. OTKA K 68612). R.C.F. acknowledges a research fellowship from the Regional Government of Andalucía.

${ }^{1}$ V. K. Pecharsky and K. A. Gschneidner, Jr., Phys. Rev. Lett. 78, 4494 (1997).

${ }^{2}$ A. M. Tishin and Y. I. Spichkin, The Magnetocaloric Effect and its Applications (Institute of Physics, Bristol, 2003).

${ }^{3}$ V. Franco, A. Conde, and L. F. Kiss, J. Appl. Phys. 104, 033903 (2008).

${ }^{4}$ V. Franco, J. S. Blázquez, and A. Conde, Appl. Phys. Lett. 89, 222512 (2006).

${ }^{5}$ V. Franco, A. Conde, J. M. Romero-Enrique, and J. S. Blázquez, J. Phys.: Condens. Matter 20, 285207 (2008).

${ }^{6}$ V. Franco, J. S. Blázquez, and A. Conde, J. Appl. Phys. 103, 07B316 (2008).

${ }^{7}$ A. Arrott and J. E. Noakes, Phys. Rev. Lett. 19, 786 (1967). 